\title{
Fate of Hydrophilic Nanoparticles in Biological Environments
}

\author{
Catherine A. Schütz ${ }^{\S \star a \#}$, Frederic Schmitt ${ }^{\mathrm{b}}$, Lucienne Juillerat-Jeanneret ${ }^{\mathrm{b}}$, and \\ Christine Wandrey ${ }^{\mathrm{a}}$
}

§SCS Poster Prize Winner

\begin{abstract}
The nanoparticles developed are based on chitosan, a biocompatible and biodegradable polysaccharide. The chitosan nanoparticles are formed in an entirely water-based process by electrostatic interactions with other biocompatible molecules. As a prerequisite to understand the fate of such nanoparticles in cells, comprehensive characterization and stability studies serve to identify quantitatively the impact of the raw material characteristics and preparation conditions on the nanoparticle characteristics. Methods included ${ }^{1} \mathrm{H}$ NMR spectroscopy, dilution viscometry, particle size analysis and electron microscopy. Cytotoxicity and cell uptake experiments on RAW 264.7 murine macrophages and p23 murine endothelial cells were performed to investigate the correlation with nanoparticle characteristics and effect of surface decoration with alginate. Cytotoxicity was assessed by the MTT survival test; cell uptake was monitored by fluorescent microscopy using labeled polymers.
\end{abstract}

Keywords: Chitosan · Electrostatic complex formation · Hydrophilic nanoparticles

\section{Introduction}

Hydrophilic nanocarriers, primarily formed by electrostatic interaction between their components, are recognized as nanoparticles having an enormous potential as vectors in biomedical and pharmaceutical applications. However, comprehensive information about the biological fate of such hydrophilic and potentially degradable nanoparticles is lacking. Due to their size, nanoparticles are able to be taken up by cells and transport molecules into cells via pathways forbidden to bigger structures of even identical chemical nature. Understanding particle-cell interactions is crucial to develop safe and successful nanocarriers for biomedical and pharmaceutical applications. ${ }^{[1]}$

In order to understand the particle-cell interactions, nanoparticles and their raw mate-

${ }^{*}$ Correspondence: C. A. Schütz ${ }^{a}$

Tel.: + 41216931737

Fax: + 41216939690

E-mail: catherine.schuetz@epfl.ch

aLaboratoire de Médecine Régénérative et

Pharmacobiologie

Ecole Polytechnique Fédérale de Lausanne

Station 15

$\mathrm{CH}-1015$ Lausanne

bInstitut Universitaire de Pathologie

Centre Hospitalier Universitaire Vaudois

Rue du Bugnon 25

$\mathrm{CH}-1011$ Lausanne

"C. A. Schütz and F. Schmitt have contributed equally to this work rial have to be characterized in detail. Process parameters should be controlled in order to establish correlations between nanoparticle characteristics and raw material properties as well as process parameters. Then the fate of the nanoparticles in biological environments can be accessed in relation to their characteristics.

This paper presents characterization results using a biocompatible polysaccharide, chitosan, as a base to form nanocarriers. ${ }^{[2]}$ The nanoparticles are formed through electrostatic interactions between chitosan and

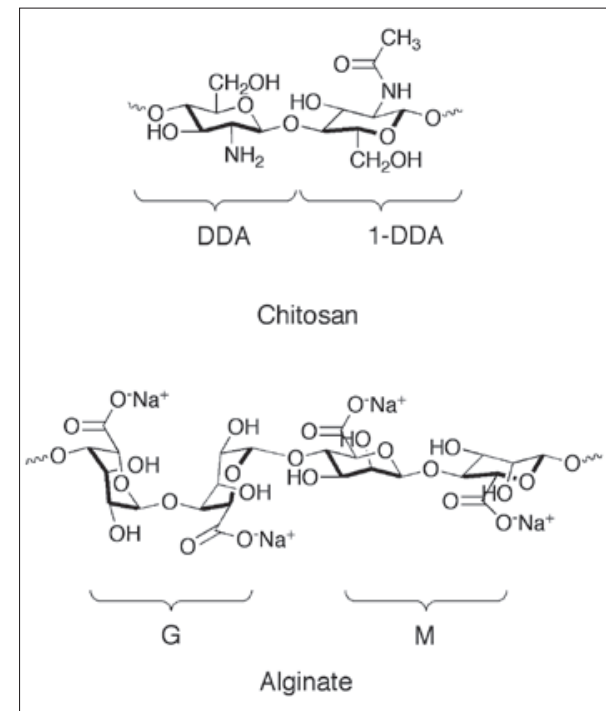

Fig. 1. Chemical structure of chitosan, a copolymer of randomly distributed $\beta-(1-4)$ linked $\mathrm{D}$-glucosamine and $\mathrm{N}$-acetyl-Dglucosamine, and of sodium alginate, a linear polysaccharide composed of $\beta$-D-mannuronic acid (M) and $\alpha$-L-guluronic acid residues. other biocompatible molecules. Chitosan is a biodegradable polyelectrolyte and one of the rare natural-based polymers which is cationic in acidic conditions (Fig. 1). ${ }^{[3]}$ Chitosan is derived from chitin by a deacetylation process. The major source of chitin is the exoskeleton of crustaceans such as crabs and shrimps, and several fungal sources, including edible mushrooms. ${ }^{[4,5]}$ In the present study, chitosans of both animal and fungal origins are used and compared. The surface of these nanoparticles is further decorated with alginate, an anionic polysaccharide. This coating allows the study of the effect of surface chemistry and charge on the fate of such nanocarriers by means of electrostatic interactions. The chitosan-based nanoparticles have great potential to be used as nanocarriers when loaded with various molecules and therapeutic agents. ${ }^{[6]}$

\section{Experimental}

\subsection{Materials}

Chitosan from crustacean shell (low viscosity chitosan, Primex, Iceland) and chitosan biotechnologically produced from Paris mushrooms (Batch $060911 \mathrm{CsUp}$ and batch L07293CsU, Kitozyme, Herstal, Belgium) were purchased. Chitosans are further denoted in this paper as chitosan $\mathrm{P}$ for the animal chitosan, and respectively chitosans $\mathrm{Ka}$ and $\mathrm{Kb}$ for the fungal chitosans. Penta sodium triphosphate solution (TPP) was purchased from Sigma-Aldrich (Sigma-Aldrich, Steinheim, Germany). Purified sodium alginate and hyaluronic acid were kindly provided by Medipol SA, Lausanne, Switzerland. 


\subsection{Methods}

\subsubsection{Chitosan Purification}

Chitosans were purified through steps of acidic dissolution, $0.1 \mu \mathrm{m}$ filtration, precipitation and dialysis.

\subsubsection{Structural and Macromolecular Characterization}

Two main characteristics of chitosan, the degree of deacetylation (DDA) and the intrinsic viscosity $([\eta])$ were analyzed for the three chitosan samples. The DDA was obtained by ${ }^{1} \mathrm{H}$ NMR spectroscopy using a Bruker Avance DPX device (400 MHz, Bruker Germany) at room temperature. Samples were dissolved with $2 \% \mathrm{DCl}$ in $\mathrm{D}_{2} \mathrm{O}$. Peak assignments and DDA determination were based on procedures previously reported. ${ }^{[7-9]}$ The intrinsic viscosity was determined by dilution viscometry using an Ubbelohde automatic viscometer, capillary $0.58 \mathrm{~mm}$, Viscologic Tl 1 (Sematech, Nice, France), equipped with an automatic dilution system. Chitosan was dissolved in acetate buffer $0.02 \mathrm{M}$ and appropriate $\mathrm{NaCl}$ concentration. The [ $\eta$ ] values were obtained according to Schulz-Blaschke and Huggins. ${ }^{[10,11]}$

\subsubsection{Nanoparticle Formation and Coating}

A $0.1 \% \mathrm{w} / \mathrm{v}$ chitosan solution was prepared by dissolving chitosan in double-distilled $\mathrm{H}_{2} \mathrm{O}$ adding $1 \mathrm{M} \mathrm{HCl}$ dropwise until the $\mathrm{pH}$ was stabilized below 4.0. This $\mathrm{pH}$ ensures complete protonation of the amine groups. The solution was left overnight at $4{ }^{\circ} \mathrm{C}$ before use. All solutions were sterile filtered prior to use. The nanoparticles were formed by adding a TPP $0.1 \% \mathrm{w} / \mathrm{v}$ solution to the chitosan solution (1:9 TPP to chitosan volume ratio) in a slow dropwise manner and strong agitation. The formulation was stirred for at least $1 \mathrm{~h}$ and stored at $4{ }^{\circ} \mathrm{C}$ overnight. For nanoparticle coating, the above formulation was diluted to $0.02 \% \mathrm{w} / \mathrm{v}$ (dry residue). An equal volume of nanoparticle dispersion was then added dropwise to a $0.05 \% \mathrm{w} / \mathrm{v}$ aqueous alginate solution under strong agitation. Finally, the $\mathrm{pH}$ was adjusted to 7.2 by adding $0.1 \%$ w/v NaOH. For cell uptake studies, chitosan was fluorescently labeled (1:50) with carboxyrhodamine (5(6) carboxytetramethylrhodamine, Fluka, Germany) using standard carbodiimide conjugation.

\subsubsection{Nanoparticle Characterization}

The surface charge of the nanoparticles was analyzed by measuring the electrophoretic mobility in a Malvern Nanosizer (Malvern, UK) to obtain the zeta potentials. The particle size distributions were studied by scanning electron microscopy (SEM) (XLF30 FEG, FEI) and transmission electron microscopy (TEM) (CM-10, Philips/FEI). For SEM imaging, nanoparticles were dialyzed $24 \mathrm{~h}$ against double distilled water and dried on a SEM aluminum support. For TEM,

Table. Degree of deacetylation (DDA) from ${ }^{1} \mathrm{H}$ NMR spectroscopy and intrinsic viscosity ([ $\left.\eta\right]$ ) and coefficients $\mathrm{k}_{\mathrm{SB}}$ and $\mathrm{k}_{\mathrm{H}}$ from dilution viscometry according to Schulz-Blaschke (SB) and Huggins $(\mathrm{H})$.

$\begin{array}{llllll}\text { Chitosan } & \text { DDA }[\%] & {[\eta]_{\mathrm{SB}}[\mathrm{mL} / \mathrm{g}]} & \mathrm{k}_{\mathrm{SB}} & {[\eta]_{\mathrm{H}}[\mathrm{mL} / \mathrm{g}]} & \mathrm{k}_{\mathrm{H}} \\ \mathrm{P} & 87 & 461 & 0.274 & 446 & 0.431 \\ \mathrm{Ka} & 92 & 225 & 0.409 & 221 & 0.555 \\ \mathrm{~Kb} & 89 & 329 & 0.305 & 324 & 0.420\end{array}$

nanoparticles were deposed on a 200-mesh carbon/formvar coated copper grid (Electron Microscopy Sciences, Hatfield, PA, USA) using the droplet method. Briefly, $10 \mu \mathrm{l}$ of nanoparticles were dropped on the grid, followed by two washings with $10 \mu$ d double distilled water and $10 \mu \mathrm{l} 2 \%$ uranyl acetate staining solution (uranyl acetate dihydrate, Sigma-Aldrich/Fluka, Germany).

\subsubsection{Cell Culture}

The RAW 264.7 murine macrophages were obtained from the ATCC (American Tissue Culture Collection, Manassas, Virginia, USA). The p23 murine endothelial cells ${ }^{[12]}$ were kindly provided by Lindsay Williams (EMBL, Heidelberg, Germany). All cell culture reagents were obtained from GibcoBRL (Basel, Switzerland). RAW 264.7 cells were grown in DMEM medium containing $4.5 \mathrm{~g} / \mathrm{l}$ glucose, $10 \% \mathrm{v} / \mathrm{v}$ heat-inactivated fetal calf serum (FCS) and penicillin/streptomycin. p23 cells were grown in DMEM medium containing $1 \mathrm{~g} / \mathrm{l}$ glucose, $10 \% \mathrm{v} / \mathrm{v}$ FCS and penicillin/streptomycin.

\subsubsection{Determination of Cytotoxicity}

Cells were grown in 96-well cell culture plates (Corning, NY, USA) until 75\% confluent. The culture media were replaced with fresh medium containing nanoparticles diluted in complete medium and the cells were exposed to the nanoparticles for 24 h. Then, medium was replaced with complete medium without nanoparticles and the cell viability was evaluated using the MTT assay (3-(4,5-dimethyl-2-thiazoyl)-2,5-diphenyltetrazolium bromide, Roche, Mannheim, Germany), essentially as previously described. ${ }^{[13]}$ Briefly, MTT, $250 \mu \mathrm{g} / \mathrm{ml}$ final concentration, was added to the cells for 2 $\mathrm{h}$, then the cell culture supernatants were removed and the cell layers were dissolved in iPrOH/0.04N HCl, absorbances at $540 \mathrm{~nm}$ were measured (iEMS Reader MF, Labsystems, Bioconcept, Switzerland) and compared to the values of control cells incubated without nanoparticles.

\subsubsection{Fluorescence Microscopy}

Cells were grown on histological slides in complete medium until $25 \%$ confluent and exposed to nanoparticles $24 \mathrm{~h}$ in the dark. At the end of the incubation period, slides were washed with PBS and nuclei were stained with 4',6'-diamidino-2-phenylindolylhydrochloride (DAPI, Roche Diagnostics, Mann- heim, Germany) in PBS according to the manufacturer instructions. Then slides were mounted in PBS and analyzed under a fluorescence microscope (Axioplan2, Carl Zeiss, Feldbach, Switzerland) with filters set at 365 $\pm 5 \mathrm{~nm}$ excitation light (BP 365/12, FT 395, LP 397) for DAPI and $535 \pm 25 \mathrm{~nm}$ excitation light (BP 510-560, FT 580, LP 590) for carboxy-rhodamine, essentially as previously described.[6]

\section{Results and Discussion}

\subsection{Chitosan Characterization}

Chitosan characterization experiments (Table) indicated a similar DDA of about $90 \%$ for all three chitosans. Such high DDA is a prerequisite for stable nanoparticles formation by electrostatic complex formation. Due to the very similar chemical composition of the samples, the intrinsic viscosity, which is related to the molar mass (MM) through the Mark-Houwink-Sakurada equation $[\eta]=\mathrm{k}_{\eta}$ $\mathbf{M}^{\alpha}$, could be used to compare the molecule sizes of the samples. The Schulz-Blaschke and Huggins coefficients $\left(\mathrm{k}_{\mathrm{SB}}\right.$ and $\left.\mathrm{k}_{\mathrm{H}}\right)$ indicated similar and good solubility of all samples. Consequently, different molar masses in the order of $\mathrm{P}>\mathrm{Kb}>\mathrm{Ka}$ were concluded for the three chitosan samples, which will allow the study of the influence of the MM on the nanoparticles formation and properties.

\subsection{Nanoparticle Properties}

For chitosan-TPP nanoparticles, comparable positive zeta potentials in the range of 33 to $39 \mathrm{mV}$ were obtained when performing the measurements in $0.9 \% \mathrm{NaCl}$ (ionic strength $154 \mathrm{mM}$ ) aqueous solutions. After electrostatic coating with alginate, the zeta potentials became negative, ranging from -45 to $-49 \mathrm{mV}$ in $0.9 \% \mathrm{NaCl}$ aqueous solutions. The shape of the nanoparticles was almost spherical with a size distribution between 50 and $300 \mathrm{~nm}$ as shown by the SEM and TEM imaging (Fig. 2, Fig. 3).

\subsection{Cytotoxicity}

Fig. 4 presents the cell survival of p23 cells after $24 \mathrm{~h}$ incubation with the nanoparticles. Chitosan $\mathrm{P}$ and chitosan Ka were compared, as well as positively (A) and negatively charged (B) nanoparticles. Although nanoparticles were mixed 1:10 with $10 x$ PBS to prevent osmotic pressure cell burst, the dilution effect is the major cause of the 


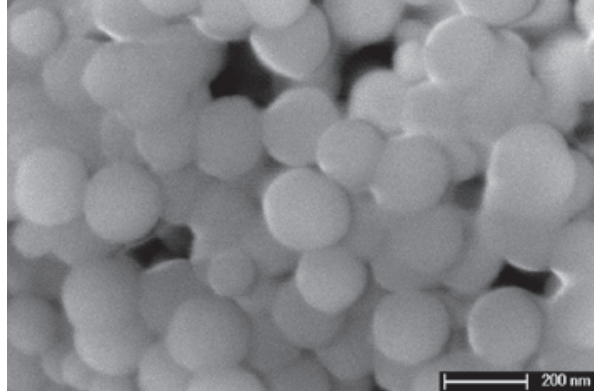

Fig. 2. Scanning electron microscopy (SEM) image of chitosan-based nanoparticles. Dried from dialyzed water-based dispersion. Tension $5 \mathrm{kV}$, working distance $8.3 \mathrm{~mm}$.

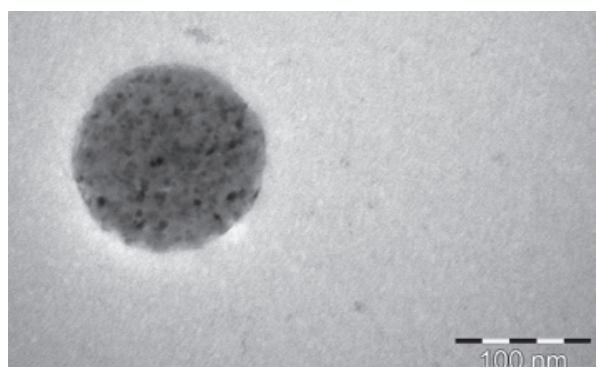

Fig. 3. Transmission electron microscopy (TEM) image of a chitosan-based nanoparticle. Dried from water-based dispersion, stained with $2 \%$ uranyl acetate solution. High-tension $80 \mathrm{kV}$, magnification 245000x.

decrease in cell survival. Indeed the decrease observed with the nanoparticles is the same as the decrease observed when only $\mathrm{H}_{2} \mathrm{O}$ $10 \%$ 10x PBS was added to the medium. Similar results were measured for both chitosan types and coated nanoparticles.

\subsection{Cell Uptake of Nanoparticles}

Cell uptake of nanoparticles prepared with fluorescently-labeled chitosan was observed under fluorescence microscopy. The concentration of $17 \% \mathrm{v} / \mathrm{v}$ was chosen since this concentration was not cytotoxic (results not shown), as previously published for this kind of nanoparticles. ${ }^{6]}$ While uncoated positively charged nanoparticles aggregated and precipitated in the cell culture media (Fig. 5 A-C), no aggregation was observed with alginate-coated nanoparticles with negatively charged surfaces (Fig. 5 D-F), indicating a higher stability in the cell culture medium probably linked to the nature of the surface charge. Moreover, the associated fluorescence of the alginate-coated chitosan nanoparticles was found in the cytoplasm but not in the DAPI-stained nucleus. This is clearly observed in Fig. $5 \mathrm{E}$ where the fluorescence spots define circles around the nuclei. Such a behavior was not observed for the uncoated nanoparticles (Fig. 5 B). This strongly suggests that the alginate-coated chitosan nanoparticles are taken up by the cell as observed with the hyaluronic acid-coated chitosan nanoparticles. ${ }^{[6]}$

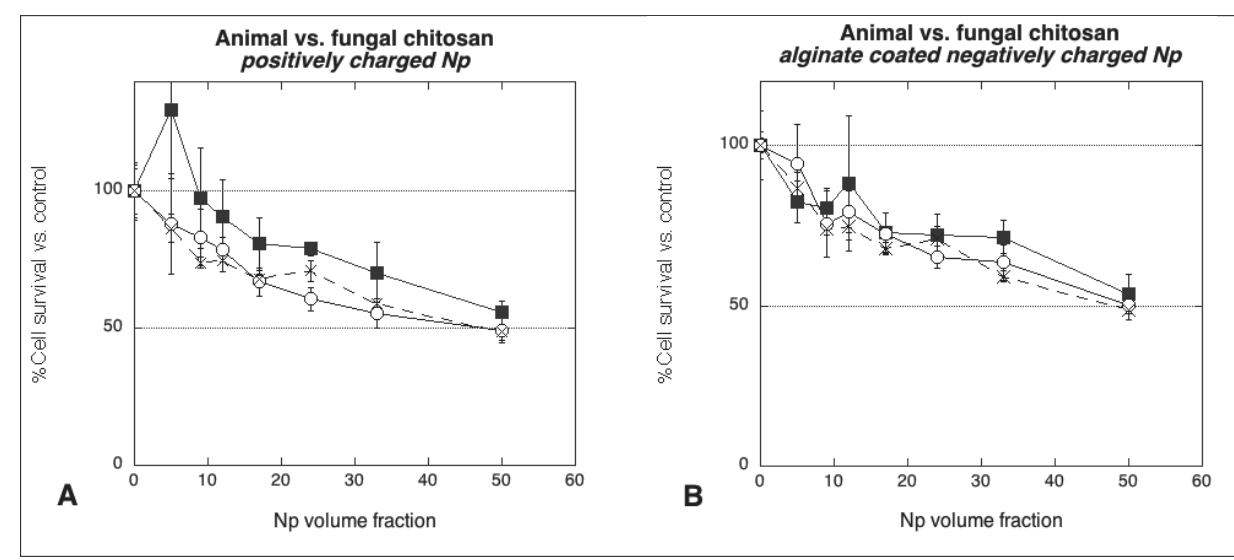

Fig. 4. Cell survival as a function of the amount of nanoparticles added to cells using the MTT assay; murine p23 immortalized endothelial cells. Chitosan P nanoparticles $\mathbf{~}$, chitosan Ka nanoparticles $\bigcirc$ and water $\times$; nanoparticles uncoated $(A)$ and coated with alginate $(B)$.
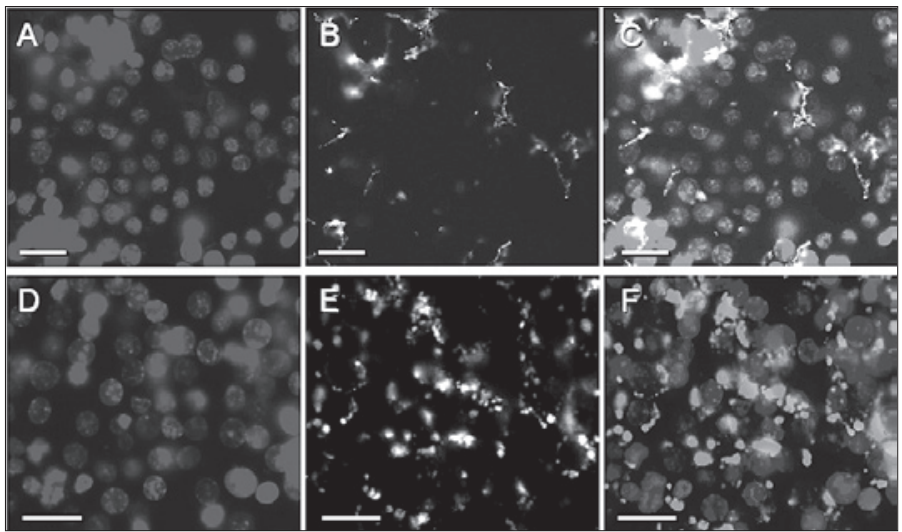

Fig. 5. Fluorescence microscopy images of RAW 264.7 cells incubated $24 \mathrm{~h}$ with $17 \% \mathrm{v} / \mathrm{v}$ of noncoated rhodamine-labeled chitosan $P$ nanoparticles $(A-C)$ and rhodamine-labeled chitosan $P$ nanoparticles coated with alginate (D-F). A \& D: DAPI staining of cell nuclei $\lambda_{\text {exc }}=365 \mathrm{~nm}$. B \& E Visualization rhodamine-chitosan $\lambda_{\text {exc }}=535 \mathrm{~nm}$. C \& F: overlays of A \& B and D \& $E$, respectively. Scale bars represent $20 \mu \mathrm{m}$ in all the pictures.

\section{Conclusion}

Structural and macromolecular characterization of three commercially available chitosan samples from animal and fungal origins were shown to have similar high DDA but different molar mass. The preparation procedure used yielded stable chitosan-TPP nanoparticles of similar size/size distribution. The zeta potentials of the raw chitosan-TPP nanoparticles were positive as expected. Further, electrostatic coating with biocompatible polyanion created negative zeta potentials for the nanoparticles. In vitro experiments demonstrated low cytotoxicity towards murine cells. Cell uptake of coated negatively charged nanoparticles was checked by microscopy and the nature of the surface charge seemed to influence the stability in cell culture media and further the uptake.

\section{Acknowledgements}

The authors acknowledge the support of the Swiss National Science Foundation (grant No 404740-117323/1), the Swiss Commission for Technology and Innovation (CTI, grant No 7985) and Medipol SA, Lausanne, Switzerland.
[1] V. Labhasetwar, Curr. Opin. Biotechnol. 2005, 16, 674.

[2] P. Kauper, C. Laue, WO2007/031812, PCT Patent application, 2007.

[3] M. Rinaudo, Prog. Polym. Sci. 2006, 31, 603.

[4] S. E. Dumitriu, in 'Polymeric Biomaterials', Ed. S. Dumitriu, CRC Press, New York, 2002.

[5] A. Steinbüchel, in 'Biopolymers', Ed. A. Steinbüchel, E. J. Vandamme, S. De Baets, WileyVCH, Weinheim, 2002.

[6] F. Schmitt, N. Rossi, L. Lagopoulos, C. Wandrey, P. Käuper, L. Juillerat-Jeanneret, J. Pharma col. Exp. Thes, 2009, submitted.

[7] M. Lavertu, Z. Xia, A. N. Serreqi, M. Berrada, A. Rodrigues, D. Wang, M. D. Buschmann, J. Gupta Ajay, Pharm. Biomed. Anal. 2003, 32, 1149.

[8] K. M. Varum, M. W. Anthonsen, H. Grasdalen, O. Smidsrod, Carbohydr. Res. 1991, 211, 17.

[9] E. Fernandez-Megia, R. Novoa-Carballal, E. Quinoa, R. Riguera, Carbohydr. Polym. 2005, 61, 155.

[10] G. V. Schulz, F. J. Blaschke, Prakt. Chem. 1941, $158,130$.

[11] M. L. Huggins, J. Am. Chem. Soc. 1942, 64, 2716

[12] L. Juillerat-Jeanneret, A. Aguzzi, O. D. Wiestler, P. Darekar, R. C. Janzer, In Vitro Cell. Dev. Biol. 1992, 28A, 537.

[13] F. Schmitt, P. Govindaswamy, G. Süss-Fink, W H. Ang, P. J. Dyson, L. Juillerat-Jeanneret, B. Therrien, J. Med. Chem. 2008, 51, 1811. 